\title{
ENTREVISTA CON AURA ELENA BERNAL DE ROJAS: DECANA DE LA FACULTAD DE EDUCACIÓN
}

Conversamos con la profesora Aura Elena Bernal de Rojas, Decana de la Facultad de Educación, conocimos sus impresiones sobre la Facultad, los profesores, los estudiantes, los grupos de trabajo, sus proyectos académicos, su pensamiento sobre el decreto 272 y otros aspectos. Presentamos esta conversación a nuestros lectores para que tengan una visión más real de la persona que orienta la educación en la Universidad Surcolombiana.

P/ Después de cuatro meses en la Decanatura ¿Cómo te sientes?, ¿Cómo sientes el trabajo? ¿Sientes que realmente es un trabajo que te hace crecer y que te permite hacer región y país?.

$\mathrm{R} /$ Me siento muy comprometida con el programa propuesto a la comunidad universitaria para que desde esta Universidad y en particular desde la Facultad de Educación contribuyamos a construir región y país. ¿Como contribuir? Siendo pertinentes con lo que hacemos a partir del conocimiento cierto sobre las realidades de nuestro sector de influencia, ubicándonos en la dinámica del desarrollo nacional y global, actuando en términos de calidad y con rigor científico, comprometidos con las comunidades, con la sociedad civil. Comprendo la dimensión de semejante compromiso, para lograrlo es necesario establecer condiciones internas como la consolidación de la comunidad académica en torno a propósitos comunes, la infraestructura necesaria, los recur- sos financieros y las relaciones de cooperación con organismos locales, nacionales e internacionales. Tal vez comencemos a ver los resultados en el mediano plazo. Sembrar los cimientos para las transformaciones esperadas cuestan mucho tiempo y dedicación. Es algo que no puedo hacerlo sola. Por fortuna ya hay una buena cantidad de profesores comprometidos en los proyectos mediante los cuales se concretará la acción de la Facultad. Creo que hay claridad hacia dónde debemos ir y sobre las rutas de cómo hacerlo.

Estoy satisfecha del trabajo que se ha adelantado hasta ahora, sobre todo porque he descubierto la riqueza de muchos de los profesores de nuestra Facultad. Su conocimiento, experiencia, espíritu de trabajo, proyectos, esperanzas, ideales... y en esta intercomunicación estoy creciendo.

Mi papel como Decana no es tanto qué produzco o qué hago, sino más bien qué y cómo lidero y apoyo, cómo gestiono y consigo los recursos para que los sueños de quienes están comprometidos con estos proyectos salgan del terreno de las idealizaciones a las realidades. La reforma la harán fundamentalmente los profesores y los estudiantes, los he visto entusiasmados, incluso a quienes parecían distantes.

P/ En estos cuatro meses ¿Cuál ha sido el escollo más dificil que has encontrado en la Facultad?. 
R/ El financiero. La dificultad de acceder a recursos. La universidad tiene un manejo centralizado, y la distribución de los recursos está en manos del Rector y los Vicerrectores. Incluso, aunque los proyectos, los equipos, los suministros están contemplados en el presupuesto no llegan o demoran mucho su entrega a las unidades. En la elaboración del presupuesto de la Universidad no participan las Decanaturas, de modo que aún no nos es claro de cuánto podemos disponer, o si los recursos que dispusieron garantizan el desarrollo de las actividades académicas como las prácticas, laboratorios, proyectos de investigación... Agreguemos a esto las dificultades financieras por las que atraviesan las instituciones del estado y por tanto las universidades, la nuestra no escapó al recorte severo que se le aplicó en el rubro de inversión que cubre estas actividades. Se insiste frecuentemente en la búsqueda de recursos por autogestión de las Facultades, pero ¿cuántas entidades están dispuestas a invertir en educación?. Ni siquiera quienes tienen este compromiso asignan los recursos a pesar de los mandatos legales establecidos en las leyes y la gestión que se ha adelantado ante estos organismos. Algo se ha hecho con los cursos de extensión, pero son cantidades muy pequeñas, nuestra población son los maestros y todos sabemos de sus precarias condiciones.

Este es un aspecto de gran preocupación, pues para adelantar los proyectos de la Facultad se necesita además del recurso humano contar con otras condiciones materiales para la financiación de los encuentros, los talleres, la producción de materiales, apoyo técnico, desplazamientos...etc. Es urgente contar con estas condiciones básicas de lo contrario se van a ver frustrados los proyectos y tal vez la esperanza de lograr la transformación esperada.

P/ ila actual crisis económica pone en peligro el desarrollo de la Facultad de Educación y el de todas las facultades o especialmente a la Facultad de Educación?
$R /$ Pone en peligro a todas las Facultades en la medida en que no se dispone de los recursos que garanticen el cabal cumplimento de las actividades académicas en curso. Pero en particular pone en peligro a la Facultad de Educación por cuánto tiene el compromiso de reformar sus programas en términos muy perentorios y en un corto plazo, prácticamente disponemos de un año para hacerlo. En este término de tiempo debemos garantizar las condiciones básicas que exige el Decreto 272, las líneas de investigación consolidadas para articular a ellas los programas de pregrado y postgrado, los recursos financieros que permitan la realización investigativa de docentes y estudiantes, los docentes dedicados exclusivamente a la investigación, el programa de formación de docentes universitarios que defina las acciones para su perfeccionamiento, las instalaciones, equipos, laboratorios y materiales exigidos según la naturaleza de cada programa, el currículo de cada programa extendido a 5 o 6 años según sea diurno, nocturno o a distancia, que incorpore integralmente la pedagogía, la investigación, el conocimiento disciplinar y la formación ética, la estructura organizativa, la legitimidad institucional de la Facultad de Educación como unidad formadora de docentes en todos los niveles y su Proyecto Educativo Institucional.. Estos requisitos deben alcanzarse en un tiempo muy corto. El no contar con los recursos básicos para hacer este trabajo nos afecta de manera muy particular.

P/ ¿En la universidad, sí hay ya, a nivel de directivos, esa voluntad para que la Facultad de Educación de la USCO pueda responder a esos requerimientos del Decreto 272 ?

$\mathrm{R} /$ Creo que sí. Al menos en este momento está aprobada la programación académica del semestre y se dieron todos los tiempos solicitados para la dedicación de los profesores a los proyectos. No hubo objeción alguna a estas dedicaciones y más bien se apoyaron las iniciativas. Faltan los recur- 
sos económicos adicionales. Los equipos de profesores están consolidando sus propuestas de trabajo con sus respectivas actividades, cronogramas y cálculos de costos. Ya hicimos un encuentro de socialización de los diferentes proyectos y se están haciendo los ajustes. Espero que se vinculen los estudiantes, los egresados y las entidades que tienen que ver con los procesos académicos de la Facultad. Presentaremos un consolidado de todos los proyectos con el calculo de los costos al Consejo Académico, a más tardar en el mes de Agosto. Espero que me sea posible convencer y obtener los recursos financieros.

P/ Con relación al Decreto 272 , realmente ¿Qué piensa la Decana con relación a ese Decreto? ¿Qué importancia tiene ese Decreto para la Facultad de Educación? ¿En qué perspectiva pone ese Decreto a la Facultad de Educación?.

$R /$ El Decreto nos pone en una situación nueva, de reflexión, de construcción, de organización. Me preocupa la demanda que puedan tener los programas al extender su duración, lo cual los pone en desventaja con respecto a los programas de formación para otras profesiones. Podría llegar a quedar esta Facultad sin estudiantes, más si encontramos poco atractivo el salario del maestro y el cada vez más reducido campo laboral, y que las Escuelas Normales Superiores tendrán la potestad de formar maestros en sólo dos años. La acreditación previa que se exige sólo a las Facultades de Educación no es voluntaria, como si lo es para las demás Facultades, es decir para carreras que forman otro tipo de profesionales. En este sentido, yo encuentro una colisión de competencias entre la Leyes 115 de 1994 y 30 de 1992. Esta última da autonomía a la universidad en desarrollo a lo establecido por la Constitución Nacional y la Ley 115 hizo una intromisión en las Universidades para quitar esta autonomía a las Facultades de Educación. Yo pienso que se está vulnerando la autonomía universitaria, que por Carta Constitucional tienen las Universidades.

Si bien es cierto que en la calidad de la educación tiene especial importancia la formación del maestro, aspecto que es necesario revisar y cualificar, también es cierto que está calidad no depende sólo de este factor, pues se conjugan las políticas nacionales, los recursos que se disponen para el desarrollo del sector, la selección y ubicación de estos profesionales, las condiciones que se brindan para el desempeño laboral, los materiales y equipos educativos a que tienen acceso, el fomento y apoyo para su formación permanente, la actitud de los maestros, el reconocimiento salarial y su reconocimiento social. Aspectos últimos sobre los cuales no se ven políticas claras por parte del estado. Bien valdría la pena que al lado de las normas expedidas para la regulación de las instituciones formadoras de docentes se dieran acciones paralelas para atender estos otros factores que pueden ser tan o más determinantes que el hasta ahora cuestionado.

Por otro lado, preocupa que el gobierno nacional expida las normas y exija su cumplimiento, sin que facilite las condiciones para su puesta en marcha. La pregunta es: con la restricción a la que han sido sometidas las universidades públicas del país, ¿dónde están los recursos para adelantar la acreditación previa de las Facultades de Educación, cómo se garantizarán los docentes para el año adicional de los programas de pregrado, cómo se financiarán los programas de formación de los profesores de las Facultades de Educación, existen los recursos que financien la investigación en educación y la asignación de docentes dedicados solamente a esta función?

Como Junta Directiva de ASCOFADE, (Asociación Colombiana de Facultades de Educación,que agrupa a más de 75 universidades del país, de la cual formo parte), tuvi- 
mos una entrevista con el Ministerio de Educación saliente, Dr. Jaime Diez Niño, en la ciudad de Santafé de Bogotá el pasado mes de Julio, y ante la pregunta de si el Gobierno Nacional había dispuesto recursos para la reforma de las Facultades de Educación, nos contestó sinceramente que NO.

Ahora, mirándonos al interior de nuestra Facultad de Educación, creo que tenemos desarrollos suficientes y fortalezas que nos permitirán salir airosos. Tenemos buen número de docentes de tiempo completo, con alto nivel de formación en su mayoría, con una trayectoria investigativa y comunitaria, y una experiencia de casí 30 años formando maestros. Es un momento oportuno para evaluarnos, para reconstruir y fortalecernos. Yo lo veo beneficioso no sólo porque exista un decreto que nos presiona sino porque nosotros mismos ya lo habiamos pensado, existía consenso en que era oportuno enrumbar nuestro norte, replantear los currículos de los programas en torno al ideal del maestro que se necesita y deseamos formar para nuestra región, igualmente las actividades de investigación y extensión para que desde la Facultad ayudáramos al mejoramiento de la calidad de vida de los huilenses. Nosotros estábamos en esta tónica, ahora también estamos siendo empujados desde afuera.

Si miramos el panorama nacional, podemos entender el motivo de esta presión, que se da con un afán regulador para frenar a aquellas instituciones universitarias que sin trayectoria académica en la formación de educadores, de la noche a la mañana se expandieron por todos los rincones del país ofreciendo programas de pregrado y especializaciones sin el menor rigor académico y en tiempos mínimos. Se dio un desbordamiento, incluso de universidades públicas, que vieron esta oportunidad rápida y fácil de captar recursos con programas de baja inversión, mediante extensión en sedes y subsedes, convenios con Alcaldias, y entre
Universidades para ahorrarse tiempo en el diseño y aprobación de los programas. En aras de la calidad, veo necesaria la medida. Es una oportunidad para que como Universidad Surcolombina y como Facultad de Educación revisemos lo que estamos haciendo en los programas de la Facultad de Educación que se están ofreciendo en precarias condiciones no sólo en nuestra casa central de estudios, sino los que se realizan en las sedes de Pitalito, La Plata, Espinal, Algeciras y Aipe, en los programas en convenio con las Universidades del Tolima, Quindio y Amazonia (Educación Física, Administración Educativa, Tecnología Educativa y Español y Comunicación ), e incluso de los postgrados en Pitalito, Doncello, Florencia, Santafé de Bogotá, Sabaneta y Quibdó. Esta evaluación nos permitirá ser más rigurosos y exigentes en lo sucesivo.

P/ Eso es un conflicto sin salida. Porque si no hay apoyos económicos, tampoco hay leyes complementarias y si hay una exigencia, eso pone en aprietos la Facultad de Educación. Frente a eso iqué políticas asumen usted como Decana de la Facultad de Educación aqui en el Huila y a nivel Nacional ASCOFADE. Los decanos qué están haciendo frente a ese Decreto que realmente pone en una situación crítica a la Facultades de Educación?

$\mathrm{R} / \mathrm{De}$ hecho, las mejores posibilidades las tienen las universidades públicas. De una u otra manera esta Universidades tienen una financiación del estado para su funcionamiento, han logrado vincular sus docentes de tiempo completo, se han preparado mediante comisiones de estudio u otras vías, han hecho investigación, tienen una presencia en las regiones y existen legalmente como unidades académicas dedicadas especialmente a la formación de educadores. Nosotros tenemos un potencial humano preparado que nos garantiza en buena parte las posibilidades de éxito.

Con relación a la posición de los Decanos de las Facultades de Educación, a través 
de ASCOFADE se han hecho pronunciamientos pidiendo que se revise el tiempo de duración de los estudios, la expedición de títulos por niveles, los subregistros para los programas en extensión, la asignación de recursos para el proceso de acreditación previa y su posterior puesta en marcha. Además ASCOFADE ha solicitado sea tenido en cuenta como interlocutor ante en Ministerio de Educación Nacional, el Icfes y el Comité Nacional de Acreditación para definir los parámetros de la acreditación previa, seleccionar los pares académicos y asesorar a las instituciones.

Por su parte, ASCOFADE se ha propuesto organizar un banco de docentes universitarios de las Facultades de Educación de todo el país para seleccionar pares académicos éticos y de altas calidades científicas que harán las respectivas evaluaciones. Desde esta instancia, se propiciarán los espacios para la reflexión y el debate de temas importantes sobre la pedagogía como eje importante del saber del maestro, los modelos curriculares, la formación disciplinar y la investigación en la educación. También se buscará el intercambio de docentes y estudiantes entre facultades y se conformará una red para pasantías, proyectos de desarrollo e investigación que permitan el crecimiento mutuo de las facultades.

En lo organizativo, se constiturán los capitulos de ASCOFADE por zonas. Se busca la unión de las Facultades de Educación para que trabajen sobre sus problemas comunes, que pueden llevarse a asambleas generales a nivel nacional. A nosostros nos corresponde el suroccidente con el Valle, Caldas, Risaralda, Quindio, Caquetá, y Tolima. Nuestra misión es organizarnos como Facultades en una red y a través de ella ofrecer programas de postgrado a nivel de maestrias y doctorados. Teniendo identificado el recurso calificado de las universidades, podremos hacer intercambios y apoyarnos en el desarrollo de proyectos académicos de importancia.
P/ Hay universidades privadas en el país, que ya han cerrado su Facultad de Educación?

$R /$ No, hay un compás de espera hasta el año entrante. Pero ya comienzan algunas de ellas a verse frenadas, porque todo nuevo programa o toda extensión de programas ya existentes deben cumplir con los requisitos de acreditación previa estipulados a partir de la vigencia del Decreto 272 que fue expedido el 11 de Febrero de este año. Muchas de ellas ya comenzaron a prepararse $y$ algunas van bien adelantadas.

P/ ¿Lo que está sucediendo en la universidad del Valle es producto de una dinámica diferente? ¿O es producto de la dinámica de la presencia del Decreto 272.

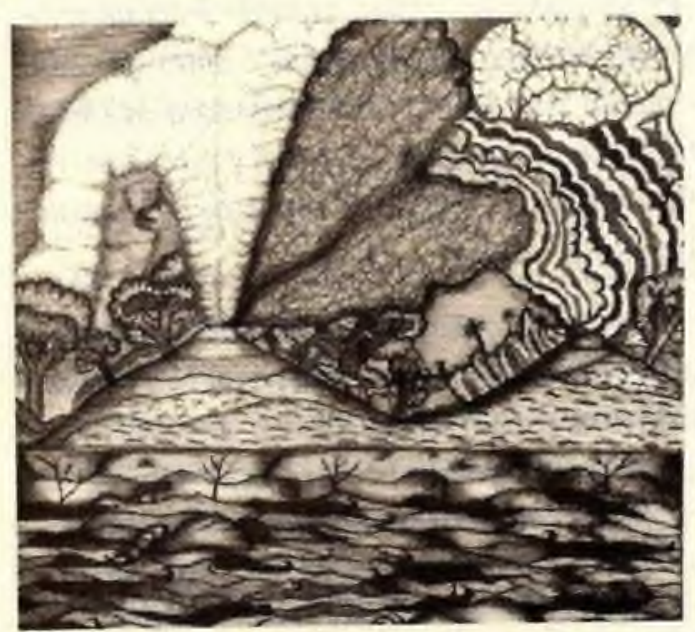

llustración: Morera C.

$\mathrm{R} /$ La universidad del Valle está pasando por una crisis que es diferente, que engloba a toda la universidad, no sólo su Facultad de Educación. Ellos tienen unos problemas de índole financiero muy graves. Tal vez se manejó muy autónoma, no racionalizó el gasto y está intervenida y creo cerrada.

La Facultad de Educación de la Universidad del Valle sufrió una transformación importante, y se reestructuró convirtiéndose en un Instituto de Investigaciones Pedagógicas, acogiéndose tal vez a la propuesta de los sabios de la Misión de Ciencia, Educación y Desarrollo, en la idea de que en el 
país existieran unos institutos de investigación pedagógica. Ellos hicieron esto, cerraron sus pregrados y se dedicaron a los doctorados, las maestrías e investigación de alto nivel.

Hablando con el Dr. Mario Diaz Villa, me decía que en este momento se ve como negativa esa decisión, porque se perdieron espacios de participación, si asisten al Consejo Académico lo hacen como invitados y las posibilidades y espacios de acción que tenían como Facultad de Educación se redujeron notablemente. Dice él quue los resultados de esta decisión posiblemente sean muy positivos y se puedan medir en un largo plazo.

$P /$ Imagino que todo cambio inicialmente produce un poco de angustia, - ¿uno no sabe que pueda suceder no? -. Volviendo a la USCO en todo este panorama nacional y este departamental interno. ¿Cómo son nuestras propuestas de trabajo?.

$\mathrm{R} / \mathrm{A}$ nivel externo, estamos buscando establecer relaciones de cooperación con las entidades gubernamentales y fortalecer nuestra acción mediante acciones de asesoría, estudio técnicos y educación continuada.

- Formamos parte del Comité Departamental de Capacitación Docente. la Facultad de Educación ha cumplido allí un papel muy importante, aportando propuestas tendientes a la organización de esta instancia, la Secretaria Técnica y la definición del Sistema de Formación Permanente de los Educadores del Huila. A través del Comité de Extensión de la Facultad de educación, en el que tienen representación todas las unidades académicas de la Facultad bajo la dirección de un docente del Departamento de Psicopedagogía, ha diseñado y ofrecido a los maestros del Huila cursos en todas las áreas. En el momento de desarrollan programas en matemáticas, informática, lengua materna y artes en los que participan 280 maestros. Es importante el convenio que se adelanta con recursos de Ministerio de Educación Nacional y el Icetex para el desarrollo de los programas : Los Maestros y los Niños Leen y Escriben y Formación en matemática para profesores de $6^{\circ}$ y $7^{\circ}$., en los que participan 660 maestros.

El Comité de extensión realizará un estudio para la Formulación de un Plan General de Formación Permanente para los Docentes del Huila, mediante el cual se determinarán las necesidades de formación y se orientarán las acciones de la Facultad de Educación.

- Participamos en la comisión del Plan Decenal del Departamento del Huila, con la vinculación de profesores de los Departamentos de Administración Educativa y Psicopedagogía. Esperamos conocer a través de ellos el estado actual de la Educación en nuestro departamento y el Plan estratégico para un periodo de 10 años.

- Somos parte del Programa de Formación de Madres Comunitarias de la ciudad de Neiva, mediante convenio con la Fundación Social, Hocol, el ICBF., La Gobernación del Huila y el Municipio de Neiva. Son beneficiarias de este programa de formación de un año, 400 madres comunitarias, y están vinculadas a él profesoras del programa de Educación Infantil.

- Colaboramos en un proyecto de evaluación de Políticas gubernamentales para el desarrollo de la infancia en el Huila, con participación de profesores del programa de Administración Educativa.

- Seguimos trabajando con las Escuelas Normales de Neiva y Gigante, se están haciendo los ajustes de los proyectos para presentarlos nuevamente al Consejo Superior de la Universidad y formalizar el convenio para la iniciación de los programas aprobados por los Consejos de Facultad y Académico. Para el efecto, ya se constituyó un Comité de Normales con participación de 
los programas de Matemáticas, Educación Infantil y Psicopedagogía.

- Para definir agendas de trabajo con el Gobierno Departamental y los Municipios se están coordinando reuniones. La primera se hará con funcionarios de la Secretaria de Educación, miembros del Consejo de Facultad y coordinadores de proyectos. Las reuniones con los Alcaldes se harán por zonas y estarán coordinada por la oficina de Planeación de la Universidad, para todas las Facultades.

A nivel interno se constituyeron los equipos y se diseñaron los siguientes proyectos que ya fueron socializados el pasado 31 de julio:

- Proyecto Educativo Institucional. Se busca a partir de la autoevaluación de las diferentes unidades académicas de la Facultad, con la participación de nuestros estudiantes, profesores, egresados y entidades que tienen que ver con la problemática educativa, definir la misión, la visión, los propósitos de la Facultad, los planes y programas, las formas de organización, los recursos requeridos y el plan de inversión, que nos permitirán sentar las bases y proyectar la transformación. Este proyecto será el eje articulador de la reforma. Será liderado por profesores del Departamento de Administración Educativa, Psicopedagogía y Matemáticas.

- Reestructuración Curricular. Buscará hacer los ajustes a todos los planes de estudio de acuerdo con los requerimientos señalados por el Decreto 272, la experiencia de la unidades académicas y las necesidades de formación de nuestros maestros. Tiene como apoyo los jefes de los Departamentos y sus Consejos de Programa, el equipo que construye el Núcleo Curricular Institucional, el equipo que está trabajando sobre el Núcleo de Facultad, la Secretaria Académica de la Facultad y el representante ante el Comité Central de Acreditación.
Lideran profesores de los Departamentos de Psicopedagogía, Lenguas Modernas y Español y Comunicación.

- Práctica Docente. Muy unido al de reestructuración curricular, este proyecto será desarrollado por el Comité de Práctica Docente de la Facultad de Educación, constituido por representantes de todos los programas y coordinado por el Departamento de Artes. El reto es trascender los procesos mediante los cuales se concreta la preparación del futuro maestro y se inicia en la enseñanza desde una perspectiva investigativa y renovadora articulada con los procesos de formación que le preceden.

- Docencia Universitaria. Diseñarán los programas de formación de docentes para la Facultad de Educación y para las demás Facultades de la Universidad.

Abordar la formación de los profesores universitarios es un reto, creemos tener la competencia para hacerlo. Se pretende alcanzar este propósito mediante cuatro acciones: Programas de inducción a profesores de reciente ingreso, Programa de Especialización en Docencia Universitaria, Programa de Maestría mediante apoyo con otras Universidades de la zona y, el Doctorado con universidades del exterior. En este proyecto trabajan profesores con formación en el campo vinculados a los programas de Educación Física y Lenguas Modernas, y a la Facultad de Ciencias Humanas y Sociales.

- Investigación. Será objeto de trabajo de Comité de Investigaciones de la Facultad, conformado por investigadores representantes de las diferentes unidades académicas, bajo la dirección de un profesor del Departamento de Matemáticas y Física, actualmente se desarrollan 20 proyectos de investigación. Buscará consolidar el Institu- , to de Investigaciones de la Facultad, mejorar los procesos investigativos, definir las líneas de investigación, articularlas con los pregrados y postgrados, con la práctica 
docente, y sentar las bases para la consolidación y expansión de comunidades académicas al interior de la Facultad y en las instituciones educativas de la región.

- Postgrados. A cargo del Comité de Postgrados, conformados por los coordinadores de estos y bajo la dirección de un profesor del programa de Administración Educativa. Tendrá este equipo la responsabilidad de hacer los ajustes curriculares para la acreditación previa de los programas de postgrado, establecer las respectivas líneas de investigación y demás requerimientos. Definir las políticas de postgrado de la Facultad, las relaciones de cooperación entre programas de postgrado y con los pregrados, los reglamentos, las proyecciones y potencialidades hacia niveles superiores de formación (maestrias y doctorados), las relaciones con las instancias de la Facultad y los programas de formación de los docentes universitarios.

- Procesos de Participación y Democratización en la Facultad de Educación. Este equipo constituido por profesores de los programas de Educación para la Democracia, Matemáticas y Física y el Departamento de Psicopedagogía, diseñará las estrategias para impulsar los procesos de participación al interior de la Universidad. Darle la participación a los estamentos para que vivan la Universidad, canalicen creativamente sus iniciativas y sientan sobre todo los estudiantes que son la razón de ser de todo cuanto hacemos.

Pensamos reactivar los comités de carrera, nombrar representantes por curso y a través de ellos dinamizar el diálogo académico que nos permita la evaluación permanente y los correctivos oportunos. Se va a proponer la rectivación del C.I.U.- Comité de Integración Universitaria. En un tiempo existió y motivó mucho al estudiantado, ellos tuvieron la oportunidad de organizar éventos académicos, culturales y recreativos. Muchos aprendieron a ser líderes.

La intencionalidad general es elaborar un proyecto macro de desarrollo académico, que articule todos los procesos de la Facultad de Educación, a través de proyectos académicos más puntuales de una unidad tan compleja, y establecer un plan económico de inversión y administración.

Ya tenemos la relación de las necesidades de todas las unidades académicas de la Facultad. Estamos haciendo los ajustes con el apoyo de la asistente administrativa y los coordinadores de los proyectos, los jefes y directores de programas y departamentos. Necesitamos cuantificar y determinar cuanto requerimos en el corto, mediano y largo plazo para definir las acciones a seguir.

P/ Hay gran cantidad de grupos y de proyectos que están trabajando, eso augura un excelente porvenir a la universidad si todo se lleva a cabo. Eso es un problema, muchas veces hay gran cantidad de proyectos y nunca aterrizan.

R/Debemos reconocer que como universidad pública tenemos un compromiso con el desarrollo de la región y que no nos podemos quedar estáticos porque las realidades son cambiantes. Tenemos que ser ágiles, concretos y pertinentes. Es preciso que despeguemos ahora y recuperemos el liderazgo que en otros tiempos tuvo la Facultad de Educación en la Universidad y en la región. Yo espero que estos proyectos lleguen a su término y que contemos con las condiciones espirituales y materiales para hacerlo. En este intento no desistiré. 